\title{
EXCEL trial—medicine turned on its head!
}

\section{Om Prakash Yadava ${ }^{1}$}

Published online: 21 November 2019

(C) Indian Association of Cardiovascular-Thoracic Surgeons 2019

We recently came out with an editorial- 'Medicine - turning on its head' looking at, 'how scientific is science?' [1]. This was a euphemism used metaphorically, but lo and behold, no time was wasted in translating it literally, thereby lending credence to our editorial. Medicine was actually turned on its head! You guessed it right- - am referring to the brouhaha and the controversy that has erupted over the EXCEL trial, raising the question, how 'NOBLE' is the 'EXCEL'?

Five-year results of the EXCEL trial, which compared percutaneous coronary intervention (PCI) versus coronary artery bypass graft $(\mathrm{CABG})$ surgery for isolated left main stenosis of low or intermediate complexity were presented at the recently held TCT meeting in San Francisco and simultaneously published in The New England Journal of Medicine [2]. These showed that risks of death, stroke or myocardial infarction (MI), which were the primary end points of the study, were identical (PCI-22.0\%; CABG-19.2\%; $p=0.13$ ). Therefore, the authors concluded that the two procedures were equivalent. However, it was surprising to find David Taggart, one of the principal investigators of the Study and the Chair of the surgical group, missing from the authors list. The mystery however cleared up soon, when he took a sling shot at the very ethos of the trial. He contended that the primary end points were changed with a view to biassing the results of the study in favour of PCI. Moreover, there was a deliberate attempt to dismiss an important mortality difference favouring $\mathrm{CABG}$, instead of making it the central focus of the paper, and in protest, he withdrew from the authorship of the trial.

In the EXCEL trial, the definition of MI included periprocedural MI also, which was biochemically and enzymatically decided and thereby designed to favour the PCI arm, right from the start. Even the very definition was tweaked towards the end of the trial's recruitment phase, and it used CKMB over cardiac troponins as the exclusive biochemical parameter, which is not

Om Prakash Yadava

op_yadava@yahoo.com

1 National Heart Institute, New Delhi, India in consonance with the second and third universal definitions of MI. In a personal communication, Taggart stated, 'The change in MI definition relates to the fact that the main protocol repeatedly states that MI will be adjudicated by the Universal Definition. In the appendix to the protocol there is a new biochemical definition of MI that had never been employed before, so this should have been a supplement to the universal definition (whose data has still not been presented) rather than replacing it'.

Speaking at the recent 33rd European Association for Cardio Thoracic Surgery (EACTS) Annual Meeting in Lisbon, Portugal, he said, 'What happened in EXCEL was a disgrace that halfway through the trial, the definition of myocardial infarction was changed. Interestingly, if you take out peri-procedural myocardial infarction, CABG is a very clear winner. I believe the data was manipulated, using a changed definition of myocardial infarction, to try and prove that for the composite end point, there is no difference (between PCI and CABG)' [3]. Taggart finds support in Marc Ruel, 'Why was the Universal MI Definition, to which every mention of MI adjudication in the 2011 protocol refers, not used to calculate the MI component of the primary end point?' [3].

It is not a matter of proverbial sour grapes (Taggart being a surgeon), for it was way back in 2018, when the EXCEL results were not known, that Taggart had in an article in 'Circulation', co-authored with Marc Ruel, prophesied that end point definition of peri-procedural MI may contribute to the 'over-optimised design and misinterpretation of these RCTs'. They further contended, 'The results of trials comparing PCI and CABG that have peri-procedural MI as a part of their composite primary end point are very sensitive to its definition' [4]. Cho et al. had earlier clearly demonstrated that definition of MI can skew the incidence of peri-procedural MI, and thereby, results of PCI versus CABG contest, dramatically ( $18.7 \%$ versus $2.9 \%$ by the second universal definition, $3.2 \%$ versus $1.9 \%$ by the third universal definition and 5.6 versus $18.3 \%$ by the Society for Cardiovascular Angiography and Interventions (SCAI) definition, respectively (Fig.1) [5].

Even Stephen Fremes from Toronto seems to agree, as the incidence of peri-operative MI in EXCEL trial was as high as 


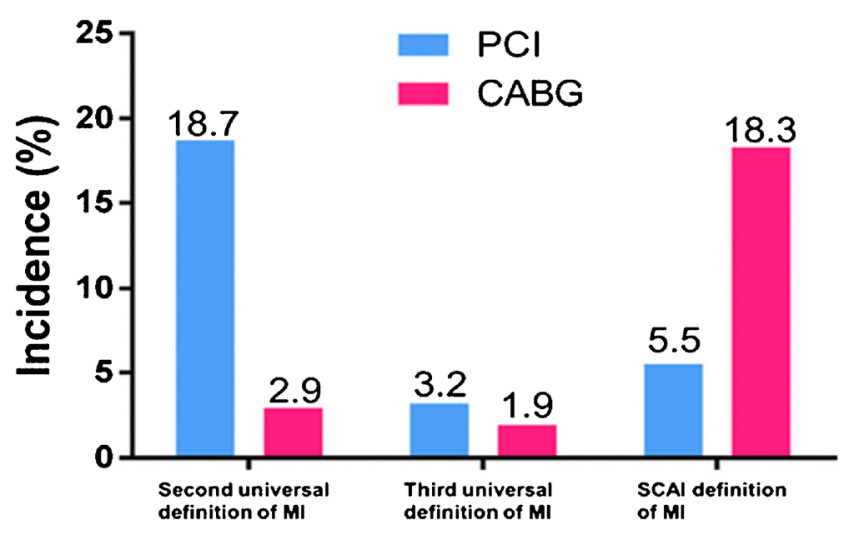

Fig 1 Differential rates of peri-procedural MI after PCI and CABG, according to various definitions [5]

$6.2 \%$ and which certainly is much more than the incidence found in recent trials such as the Freedom trial (1.7\%) and the SYNTAX trial (2.9\%). Its higher incidence therefore is more likely to be related to the biochemical definition of MI, which clearly favours PCI. Further speculating at a plausible reason for inclusion of peri-procedural MIs in primary end point, he said, '...to meet the sample size with a smaller number of patients, they wanted to have more events, and this was one of the ways of doing it'. Of note, at least one other EXCEL trialist, the chair of its, 'Statistical Committee' also expressed reservations about the inclusion of peri-procedural MI within the composite end point [3].

Second bone of contention is the import of peri-procedural versus spontaneous MIs in the follow-up period. The argument that the former are as important as the latter falls flat in the face of clear evidence available in literature that it is nonprocedural MIs in the follow-up period which are mortality generating, rather than the peri-procedural minor elevation of enzymes. Taggart emphasised that it were not the non-procedural, spontaneous MIs which were higher with CABG (to the contrary, they were higher with $\mathrm{PCI}$ ), but it were the peri- procedural MIs, which were higher in $\mathrm{CABG}$, which tweaked the results in favour of PCI.

In further analysis of mortality, it was found that death from any cause was significantly higher with $\mathrm{PCI}$ as against $\mathrm{CABG}$ at 5 years $(13.0 \%$ versus $9.9 \%$, respectively, odds ratio-OR $1.38 ; 95 \%$ confidence interval-CI $1.03-1.85 ; p=0.002$ ) (Fig. 2). The lead author of the EXCEL study, Dr. Gregg Stone, agreed that mortality was higher in PCI arm, but he down played this by saying that EXCEL trial was not powered for mortality, which argument looks rather weak. It further appeared that the mortality benefit for surgery seemed to be widening with time (Fig.2). As always, ischaemia-driven revascularisation was more frequent after PCI than after CABG (16.9\% versus $10.0 \%$; $95 \%$ CI 3.7 to 10.0$)$.

When a suggestion was made for a longer follow-up as the events and mortality were diverging at 5 years, Abbott Vascular refused for funding any further beyond 5 years, possibly and arguably fearing the long-term outcomes, which are written large in current data.

Even the direct participation of the industry in the trial and the inclusion of Chief Medical Officer (CMO) of Abbott Vascular and CMO of Medtronic as primary authors of the study is, to say the least, irregular. Taggart alleged that one third of study authors received personal fees from Abbott Vascular, while more than $40 \%$ received fees from other stent manufacturers, which speaks volumes of the authenticity of such trials. 'Ruel too took EXCEL to task for using a custom peri-procedural MI definition, one favourable to the group treated with a technology provided by the sponsor' (Abbott Vascular) [3].

Lastly, the role of the reviewers and the Editor-in-Chief who failed to pick up these issues before they decided to publish the paper has also been pointed out by Rita Redberg, herself the Editor-in-Chief of JAMA Internal Medicine. In fact, the same had been brought out in the above-said editorial of us in the Indian Journal of Thoracic and Cardiovascular
Fig. 2 EXCEL trial - death from any cause at 5 years [2]

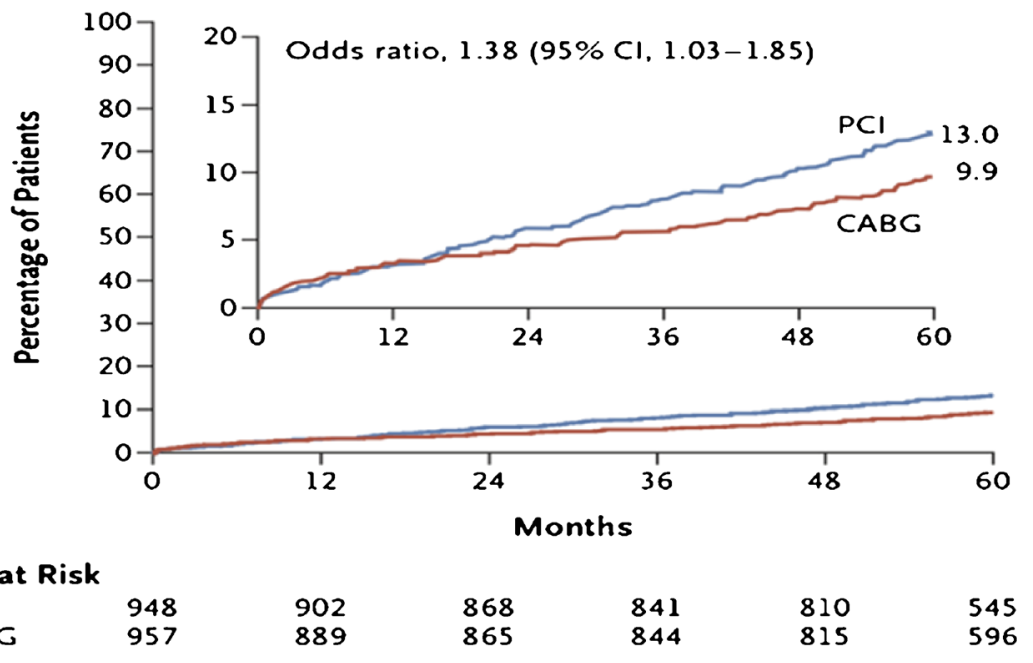


Surgery [1] and raises serious ethical questions on the standards of peer reviewership and the role of Editor-in-Chief of journals. She said, 'I cant help but see it as a failure of the review and editorial process to allow a final paper, which is clearly a disservice to patients. We are talking about a $38 \%$ difference in mortality (between PCI and CABG at 5 years).'

No wonder then, Taggart pleaded at the recent EACTS meeting in Lisbon, that the European Society of Cardiology/ EACTS guidelines granting currently a class IA recommendation for PCI in left main with a SYNTAX Score of 0 to 22 should be downgraded in view of the new findings. I am sure the forth coming debate on EXCEL trial between David Taggart and Gregg Stone at the International Coronary Congress in New York, in December 2019, is likely to be fiery and is being keenly looked forward to.

Going back to where we started-"How scientific is Science? No Brainer-Science is convoluted and has a potential to be tweaked to suit the sponsor. It's a damning and terse statement, but then, reality cannot be brushed under the carpet. It is an open secret that a large number of major clinical trials go missing, especially if they are negative. In a recent survey, Tatsioni et al. found that 67 of the 500 large randomised trials registered with ClinicalTrials.gov did not publish their results for a median of 9 years post-completion. The total number of patients involved was a staggering 87,883 [6]. No reasons were forthcoming. Harlan M Krumholz, Editor-in-Chief of NEJM Journal Watch (Cardiology) commented, 'The sharing of results is the responsibility of all scientists - and is our ethical obligation to the participants. It is far past time to address this situation and restore trust in clinical science'. He goes a step further in calling it an 'Academic misconduct' [7].

Onus therefore is on the medical fraternity to not allow any tweaking or manipulation of science. In fact, it is now high time that it is mandated for all data to be in public domain. When I talk of data, I mean raw, patient level data, as statistical analysis, can be wilfully manipulated to vitiate findings. 'The way the problem is addressed or the scientific enquiry framed, by design or default, may influence the end results' [8].

Medicine today is industry-driven, and most, if not all, research is sponsored. We must therefore learn to read between the lines of published data. Simultaneously, as a scientific community, we are being watched, so we need to be bit of a 'Hawk' - always on the guard. In our endeavour to aid industry in selling stents, we must not inadvertently sell our conscience.

\section{References}

1. Yadava OP. Medicine - Turning on its head! Editorial. Indian $\mathbf{J}$ Thorac Cardiovasc Surg. 2019;35:431-432.

2. Stone GW, Kappetein AP, Sabik JF, et al. Five-year outcomes after PCI or CABG for left main coronary disease. N Engl J Med 2019; 381:1820-1830. https://doi.org/10.1056/NEJMoa1909406

3. Sparks, Accusations fly over conduct of EXCEL PCI Vs CABG trial. Medscape. Oct 15, 2019.

4. Ruel M, Falk V, Farkouh ME, et al. Myocardial revascularisation trials - Beyond the printed word. Circulation. 2018;138:2943-2951.

5. Cho MS, Ahn JM, Lee CH, et al. Differential rates and clinical significance of periprocedural myocardial infarction after stenting or bypass surgery for multivessel coronary disease according to various definitions. JACC Cardiovasc Interv. 2017;10:1498-1507.

6. Tatsioni A, Karassa FB, Goodman SN, Zarin DA, Fanelli D, Ioannidis JPA. Lost evidence from registered large long-unpublished randomized controlled trials: a survey. Ann Intern Med. 2019;171: 300-301.

7. Wallach JD, Krumholz HM. Not reporting results of a clinical trial is Academic Misconduct. Ann Intern Med. 2019;171:293-294.

8. Yadava OP. CABG versus PCI - 'evidence' and 'practice' not the flip side. Indian J Thorac Cardiovasc Surg. 2018;34:S195-197.

Publisher's note Springer Nature remains neutral with regard to jurisdictional claims in published maps and institutional affiliations. 